\title{
Deciding with the eye: How the visually manipulated accessibility of information in memory influences decision behavior
}

\author{
Christine Platzer • Arndt Bröder • Daniel W. Heck
}

Published online: 12 November 2013

(C) The Author(s) 2013. This article is published with open access at Springerlink.com

\begin{abstract}
Decision situations are typically characterized by uncertainty: Individuals do not know the values of different options on a criterion dimension. For example, consumers do not know which is the healthiest of several products. To make a decision, individuals can use information about cues that are probabilistically related to the criterion dimension, such as sugar content or the concentration of natural vitamins. In two experiments, we investigated how the accessibility of cue information in memory affects which decision strategy individuals rely on. The accessibility of cue information was manipulated by means of a newly developed paradigm, the spatial-memory-cueing paradigm, which is based on a combination of the looking-at-nothing phenomenon and the spatial-cueing paradigm. The results indicated that people use different decision strategies, depending on the validity of easily accessible information. If the easily accessible information is valid, people stop information search and decide according to a simple take-the-best heuristic. If, however, information that comes to mind easily has a low predictive validity, people are more likely to integrate all available cue information in a compensatory manner.
\end{abstract}

Keywords Decision making · Memory · Spatial attention · Accessibility $\cdot$ Visual salience

Imagine that you are shopping for breakfast cereal. As you reach the supermarket shelf, you are stunned by the multitude

C. Platzer · A. Bröder · D. W. Heck

School of Social Sciences, University of Mannheim,

Mannheim, Germany

C. Platzer $(\bowtie)$

Experimental Psychology, University of Mannheim, Schloss EO,

68131 Mannheim, Germany

e-mail: chrmplatzer@gmail.com of different products, ranging from healthy-looking granola to fancy peanut butter oatmeal. You hesitate for a moment, and then grab a package of oat cereals. Back home you wonder why you have chosen this product and not one of the presumably tasty cornflakes.

Sometimes we deliberately control which stimuli in our environment we pay attention to. This kind of attentional control is assumed to be goal-driven and based on top-down processing (Egeth \& Yantis, 1997; James, 1890). Sometimes, however, our attention is captured by a stimulus in a bottomup fashion, irrespective of the current goals (Itti \& Koch, 2000, 2001; Parkhurst, Law, \& Niebur, 2002; Parkhurst \& Niebur, 2004). For example, studies addressing the "pop-up effect" have shown that salient stimuli can capture attention involuntarily (Pashler, 1988; Theeuwes, 1991, 1992). Marketing takes advantage of these effects. For instance, companies use unusual packages that "stick out" to increase the recognition value of their brand (Kardes, 1999). Chandon and Wansink (2002) manipulated the visual salience of different convenience goods by varying the product placement (top shelf vs. lower shelf) or the color contrast of the label (high vs. low contrast). As expected, salient products were more likely to be consumed than less salient products. Accordingly, you might have chosen oat cereals because of their eye-catching package.

Another line of research expanded this approach from an object level (decision objects vary with regard to their salience) to a feature level (certain pieces of information about decision objects are more salient than other information). In a preference task by Mandel and Johnson (2002), participants had to choose between different products, such as cars. Their results indicated that participants who were primed with a certain feature (e.g., safety vs. price) preferred those products that excelled on the respective feature. These results were replicated in judgment tasks in which participants had to estimate, for example, the price of different products (Shah 
\& Oppenheimer, 2007). Perceptually fluent information was shown to affect judgments more strongly than disfluent information. These studies provide another explanation for your decision to purchase oat cereals: You might have chosen them because a certain feature on the package (e.g., a sign saying "low sugar content"), but not the product as such, attracted your attention.

In these studies - as well as in most studies within the field of judgment and decision making-relevant information is provided at the time the judgment or the decision has to be made. This is surprising, given that many everyday decisions are based on information that has to be retrieved from long-term memory (e.g., whether we enjoyed particular cereals in the past). The present study is concerned with memory-based decisions and investigates how the accessibility of information in memory affects decision behavior. The previously mentioned studies suggested a main effect of visual salience on decision behavior: Salient products were preferred over less salient products (Chandon \& Wansink, 2002), and salient features determined which product was chosen (Shah \& Oppenheimer, 2007). Recent findings, however, have challenged this assumption. In a multi-cue inference task, Platzer and Bröder (2012) showed that decision behavior was determined by the interaction of visual salience and the predictive quality of features. More precisely, individuals used different decision strategies, depending on whether salient information allowed for making good decisions. This finding, which is also referred to as the salience effect, suggests that your choice in the introductory example might have been influenced by information that came to mind easily (e.g., whether you enjoyed the product in the past). However, the way that this information is integrated into a judgment (i.e., the decision strategy that is used) depends on its predictive quality. The aim of the following experiments was to show that the salience effect is just a special case of a broader phenomenon. Perceptual salience is only one determinant of accessibility, but other means of reducing retrieval costs have not yet been explored. We will provide evidence that different manipulations of the accessibility of information affect strategy selection in the same way.

\section{Decision strategies in multi-cue inferences from memory}

Individuals can make decisions by using information associated with the decision criterion. Going back to our example, you might have been interested in choosing the healthiest cereal that is available in the supermarket (decision criterion). Sugar content or concentration of natural vitamins might serve as cues to predict healthiness. The characteristic on a certain cue (e.g., high or low sugar content) is called a cue value. All cues are probabilistically related to the decision criterion. They vary, however, in their predictive validity. ${ }^{1}$ Whereas the sugar content of a food item is highly correlated with healthiness, the predictive power of protein content is probably lower. Since the decision can be right or wrong (e.g., if you choose less healthy cereals), it is also referred to as a multi-cue inference decision.

In order to make a choice, decision makers have to integrate the available cue information into a judgment and compare different decision objects (e.g., different cereals) against each other. Researchers have proposed a multitude of strategies for doing so (Gigerenzer \& Todd, 1999; Payne, Bettman, \& Johnson, 1993). One crucial distinction is based on the classification into noncompensatory and compensatory strategies. Noncompensatory strategies, such as the take-thebest heuristic (TTB; Gigerenzer \& Todd, 1999) are considered to be fast and frugal, since they incorporate just a subset of the available information. TTB searches cues in the order of their predictive validity starting with the most valid cue. If this cue discriminates between objects (e.g., sugar content is high for product $\mathrm{A}$ and low for product $\mathrm{B}$ ), a decision is made (e.g., product $B$ is chosen). If the most valid cue does not discriminate between objects, the second most valid cue is considered, and so on. Such simple heuristics have been shown to be as accurate as more complex decision strategies in several environments (Gigerenzer \& Goldstein, 1996). Note that TTB's search rule assumes a deliberate retrieval of cues in the sequence of decreasing cue validity. The stopping rule ends retrieval when a discriminating cue is found, and the decision rule goes with this discriminating cue. Only the compound of search rule, stopping rule, and decision rule is referred to as TTB.

In contrast, compensatory strategies take all available cue information into account. The "weighted additive rule" (WADD) and the "equal weight rule" (EQW) make a choice by summing up cue values and choosing the object with the largest sum. Whereas WADD weights cue information differently (e.g., according to cue validities), EQW uses uniform weights.

Following the idea that people possess a repertoire of strategies to choose from, one crucial question arises: How do individuals select from their repertoire of decision strategies? Several approaches have been proposed to solve this strategy selection problem. According to an effort-accuracy approach, individuals select the strategy from their repertoire that is easy to apply and, at the same time, as accurate as possible (Beach \& Mitchell, 1978; Payne, Bettman, \& Johnson, 1988, 1993). Other approaches are based on reinforcement learning. For example, strategy selection learning theory proposes that

\footnotetext{
${ }^{1}$ We use the term cue validity in a broader sense here than, for example, in the theory of probabilistic mental models (Gigerenzer, Hoffrage, \& Kleinbölting, 1991), in which it is measured as the conditional probability of a criterion value given a cue value. In the broader, Brunswikian sense, the validity of a cue is its statistical relation to a judgment criterion that can be measured by various contingency indices.
} 
people select the strategy that they expect to solve the decision problem best. Expectancies, in turn, are assumed to be modified via reinforcement learning (Rieskamp \& Otto, 2006). A promising complementary approach was recently proposed by Marewski and Schooler (2011). The cognitive niches framework states a two-stage process of strategy selection: (1) in the first stage, the number of available strategies is restricted by the cognitive system and the environment. Only a subset of strategies can actually be applied in a certain situation (referred to as a cognitive niche). (2) If different strategies are applicable, strategy selection in the second stage depends on effortaccuracy trade-offs, learning, or other selection mechanisms.

In sum, strategy selection has been shown to depend on several factors, such as the environmental payoff structure (Bröder, 2003; Bröder \& Schiffer, 2006a; Payne et al., 1988; Rieskamp \& Hoffrage, 1999), time pressure (Payne et al., 1988; Rieskamp \& Hoffrage, 2008), and cue costs (Bröder, 2000; Newell \& Shanks, 2003; Newell, Weston, \& Shanks, 2003). More importantly, however, strategy selection has been shown to depend on whether decisions have to be made from givens or from memory (Hastie \& Park, 1986). With decisions from givens, cue information is directly available at the time of decision making. You can, for instance, compare different cereals by having a look at the nutrition facts reported on the back of the packaging. Sometimes, however, cue information has to be retrieved from memory (e.g., when your husband has thrown away the packaging of your cereals to save space in the kitchen shelf). Simple heuristics such as TTB were used more often if cue information had to be retrieved from memory than if information was presented at the time of decision making (Bröder \& Schiffer, 2003). Gigerenzer and Todd (1999) argued that people used simple heuristics to limit information search in memory, which is assumed to be cognitively demanding. On the basis of these results, we investigated whether the accessibility of information in memory affects decision behavior.

\section{Manipulating the accessibility of cue information}

The aim of this article is to show that people use different strategies for solving memory-based decisions, depending on whether valid or invalid cue information can be easily accessed. In the following, we will describe two methods to perceptually manipulate the accessibility of cue information in memory. The first method was used in a study by Platzer and Bröder (2012). The authors' initial aim was to test an alternative explanation for the finding that people used different decision strategies in multi-cue inferences depending on whether cues were presented verbally or pictorially (Bröder \& Schiffer, 2003, 2006b). By showing that this format effect only occurred when less valid pictorial cues were particularly visually salient (i.e., the alleged format effect was rather a salience effect), they clearly demonstrated that strategy selection in multi-cue inference tasks is influenced by the accessibility of cue information.

In this article, we will introduce a second, newly developed method to manipulate the perceptual accessibility of verbal cue information. The results will show whether the salience effect found with pictorial cues can be generalized to verbal cues that differ in accessibility. By showing that salience is but one means of affecting retrieval amongst others, different accessibility effects might be explained under a common theoretical umbrella, such as an effort-accuracy approach (Beach \& Mitchell, 1978; Payne et al., 1988, 1993).

\section{Visual salience of pictorial cue information}

Bröder and Schiffer $(2003,2006 b)$ compared verbal with pictorial cues, and they showed that in a verbal cue condition most participants relied on TTB, whereas in a pictorial cue condition, compensatory strategies were more frequently used. They argued that this format effect occurred because pictorial cues were retrieved more easily, thus allowing for a compensatory cue integration. Platzer and Bröder (2012), however, showed that in these studies cue validity and cue salience were confounded, and that the alleged format effect was rather a salience effect.

In all of these studies (Bröder \& Schiffer 2003, 2006b; Platzer \& Bröder, 2012), participants had to solve an invented criminal case. A famous singer had been killed, and participants had to find out which of his former girlfriends was most likely to have committed the murder. Eyewitnesses had observed the fleeing perpetrator and could remember her clothing. Accordingly, participants could use information about the suspects' clothing (types of coats, tops, trousers, and bags) as cues for judging the probability of being the murderer. The experiment consisted of two critical phases: (1) In a first learning phase, participants learned by heart which kinds of clothing the suspects had worn. Afterward, they were informed about the kinds of clothing the perpetrator had worn according to the statements of the eyewitnesses. Cue validity was manipulated via the number of witnesses who agreed on a certain piece of clothing. (2) In a subsequent decision phase, two suspects were presented, and participants had to decide which suspect was more likely to have committed the crime. During this phase, all previously acquired knowledge about cue values (i.e., articles of clothing) and cue validities had to be retrieved from memory.

Platzer and Bröder (2012) used a 2 (cue format: verbal vs. pictorial) $\times 2$ (congruency of validity and salience: congruent vs. incongruent) between-subjects design to test their salience hypothesis. In conditions with a verbal cue format, information about the suspects' clothing was presented verbally. In conditions with a pictorial cue format, pictures of suspects were 
shown. In a pilot study, Platzer and Bröder determined the visual salience of the pictorial cues. In the congruent pictorial condition (CP), cue validities were assigned to the four cues, such that the validity and the salience of cues were positively correlated. In the incongruent pictorial condition (IP), salience and validity were negatively correlated.

In line with the salience hypothesis, participants in the verbal conditions, as well as those in the $\mathrm{CP}$ condition, predominantly used TTB. In the IP condition, in which less valid cues were particularly salient, participants were more likely to integrate all available cue information in a compensatory manner. Two main conclusions can be drawn from these results: First, no evidence supported the idea that pictorial cues per se could be retrieved more easily. The alleged format effect suggested by Bröder and Schiffer $(2003,2006 b)$ was rather a salience effect. Secondly, decision behavior seemed to be influenced by which information could be easily retrieved.

Visual salience is just one way of manipulating accessibility. In addition, varying the visual salience of cues might not only have affected the accessibility (i.e., how easily information could be retrieved from memory), but also the availability (i.e., whether information could be retrieved at all) of cue information in memory (Tulving \& Pearlstone, 1966). Since visual salience is an inherent property of the stimulus material, pictorial cues in the Platzer and Bröder (2012) study differed in visual salience already during the learning phase. On the basis of research demonstrating a positive correlation between an object's salience and recall performance (Fine \& Minnery, 2009; Olson, 2002), salient cues might have been memorized more deeply and could therefore be better recalled than less salient cues. To test the generalizability of the salience effect, we developed a method to manipulate accessibility during retrieval (i.e., during the decision phase). The method described in the next paragraph has the potential to investigate accessibility effects that are independent of availability effects.

\section{Spatial cueing of verbal cue information}

Visual salience is one possibility to manipulate the accessibility of cue information by enhancing encoding quality. Information that receives much attention is assumed to be encoded more deeply than is information that receives only incidental attention (Dougherty, Gronlund, \& Gettys, 2003; Logan, 1988). To manipulate the accessibility at retrieval, we took advantage of research demonstrating a link between spatial attention and memory retrieval. We strove to enhance the accessibility of certain information in memory by directing a person's attention to the spatial position on screen at which this information had been learned previously. This idea is based on the combination of two well-known phenomena: the looking-at-nothing phenomenon (Altmann, 2004; Richardson \& Spivey, 2000) and the spatial-cueing paradigm (Posner, 1980; Posner, Snyder, \& Davidson, 1980). The looking-at-nothing phenomenon describes the following finding: If people have to recall information, they tend to look at the now-blank location at which the information was previously presented. By using the location as a retrieval cue, looking at nothing facilitates memory retrieval for information associated with this location (Ferreira, Apel, \& Henderson, 2008). Using a decision task, Renkewitz and Jahn (2012) provided evidence that gaze behavior can validly indicate which piece of information people try to retrieve.

We argue that if gaze behavior can indicate the search for information in memory, information search in memory can be influenced by guiding gaze behavior. This argument is an extension of the spatial-cueing paradigm: Paying attention to spatial locations facilitates the processing of stimuli located in those locations (Posner, 1980; Posner et al., 1980). Whereas the spatial-cueing paradigm refers to situations in which information is presented on screen, we expanded this idea to situations in which information has to be retrieved from memory. Accordingly, we refer to our method as the spatialmemory-cueing paradigm (SMC).

The assumption that eye movements to "nothing" indeed increase the accessibility of certain information was recently supported by Johansson (2013). He imposed different eye movements on participants during test, which, in turn, influenced the retrieval performance of visuospatial information. The author concluded that eye movements can act as facilitatory retrieval cues, and are thus functional for retrieval rather than just an epiphenomenon.

In previous experiments, in which accessibility was manipulated via visual salience of pictorial cues, accessibility and availability effects were probably confounded. With the SMC, differences in decision behavior cannot be attributed to differences in encoding quality of cues.

\section{Present study}

We were interested in whether individuals integrate easily accessible information into their judgments. According to the salience effect (Platzer \& Bröder, 2012), we expected strategy selection to depend on the interaction between cue accessibility and validity. If valid cue information came to mind easily, the retrieval would not interfere with TTB's deliberate search order, and we expected people to stop information search and to rely on TTB. However, if less valid information came to mind easily and involuntarily, the question would arise whether-and if so, how - people integrated easily accessible invalid cues into their judgments. Three predictions would be possible: (1) People might just ignore easily accessible invalid cues and commence 
with TTB's deliberate search order according to decreasing validity of the cues. If this was true, we would expect people to keep on using TTB. On the basis of Platzer and Bröder's (2012) results, however, we argue that ignoring easily accessible information is even more cognitively demanding than integrating it into the judgment. (2) Another possibility is that people would dispense with deliberate search and exclusively rely on cue information that could be accessed easily, regardless of its validity. For example, a corresponding noncompensatory strategy might search cue information sequentially according to its accessibility rather than its validity. Following the idea that people possess a multitude of domain-specific strategies to choose from, such a strategy should be included in the "adaptive toolbox" framework (e.g., Gigerenzer \& Todd, 1999) if we were to find empirical evidence for it. However, from an effortaccuracy perspective (Beach \& Mitchell, 1978; Payne et al., 1988, 1993), deciding solely on the basis of invalid information seems unreasonable. Although such a noncompensatory strategy could be applied effortlessly, it would not allow for making accurate predictions. (3) Therefore, we hypothesized that easily accessible information is more likely to be incorporated into the judgment in a compensatory manner. The following experiments test these predictions by varying the accessibility of verbal cue information via the aforementioned SMC.

\section{Experiment 1: Manipulating accessibility in a between-subjects design}

Platzer and Bröder (2012) showed that individuals decided according to TTB if the validity and the visual salience of pictorial cues were positively correlated. In contrast, compensatory strategies were used more often if validity and visual salience were negatively correlated. Experiment 1 closely resembles the Platzer and Bröder study with respect to the materials, procedure, and the rationale underlying the experiment. Instead of manipulating the accessibility of cues via visual salience, we adopted the SMC. In Experiment 1 , we aimed at showing that the effects can be replicated (1) with verbal materials and (2) if accessibility was manipulated during retrieval, and therefore independent of encoding quality.

\section{Method}

\section{Participants}

A group of 65 students from the University of Mannheim volunteered in Experiment 1 (28 females, 37 males; mean age 22 years, $S D=3.90$ ). The data of two students had to be excluded from further analyses because they had already participated in a similar study, including postexperimental debriefing. Participants received either course credit or $€ 5$ per hour, plus a performance-contingent payment.

Materials and design

As in previous studies (Bröder \& Schiffer, 2003, 2006b; Platzer \& Bröder, 2012), we used the cover story of an invented criminal case. Participants had to decide which of ten female suspects was more likely to have killed a famous singer. Since eyewitnesses could remember the clothing of the perpetrator, different articles of clothing that the suspects had worn served as the cues. Suspects differed with regard to their types of coat (leather jacket, cardigan, blazer), top (polo shirt, shirt, blouse), trousers (jeans, leggings, linen trousers), and bag (tote bag, hand bag, leisure bag). The distribution of cue values among the ten suspects was chosen such that the different decision strategies (TTB, WADD, and EQW) made different predictions in the decision phase, which is a prerequisite for classifying decision strategies. Table 1 shows the distribution of cue values among the suspects, and Table 2 provides examples of combinations of cue patterns that allow for discriminating the different decision strategies. Cue validity was manipulated via the number of witnesses who agreed on a certain cue. For instance, the more witnesses who agreed that the suspect had worn a leather jacket, the higher was the predictive validity of the cue "coat." The critical cue value per cue and the validity hierarchy of cues were determined at random for each participant.

Cue accessibility was manipulated in a between-subjects design. In the congruent verbal condition (CV), we increased the accessibility of the most valid cue. In contrast, the least valid cue was easily accessible in the incongruent verbal condition (IV).

Table 1 Distribution of cue values among objects, adopted from Bröder and Schiffer (2003, 2006b)

\begin{tabular}{lllll}
\hline Cue Patterns & Cue 1 & Cue 2 & Cue 3 & Cue 4 \\
\hline Pattern 1 & 1 & 1 & 0 & 0 \\
Pattern 2 & 1 & 0 & 1 & 1 \\
Pattern 3 & 1 & 0 & 1 & 0 \\
Pattern 4 & 1 & 0 & 0 & 1 \\
Pattern 5 & 1 & 0 & 0 & 0 \\
Pattern 6 & 0 & 1 & 1 & 1 \\
Pattern 7 & 0 & 1 & 1 & 0 \\
Pattern 8 & 0 & 1 & 0 & 1 \\
Pattern 9 & 0 & 0 & 1 & 1 \\
Pattern 10 & 0 & 0 & 1 & 0 \\
\hline
\end{tabular}

1 denotes a critical cue value, and 0 denotes a noncritical cue value. 
Table 2 Examples of combinations of cue patterns that were presented in the decision phase of Experiment 1, and choice predictions of the strategies

\begin{tabular}{|c|c|c|c|c|c|c|c|c|c|c|}
\hline \multirow[b]{2}{*}{ Condition } & \multirow[t]{2}{*}{ Item Type } & \multirow[t]{2}{*}{ Cue Pattern } & \multicolumn{4}{|c|}{ Cue Values } & \multicolumn{4}{|c|}{ Predictions } \\
\hline & & & Cue 1 & Cue 2 & Cue 3 & Cue 4 & ТTВ & WADD & EQW & TTS \\
\hline \multirow[t]{6}{*}{$\mathrm{CV}$} & 1 & A & 1 & 1 & 0 & 0 & $\mathrm{~A}$ & A & $\mathrm{A}$ & - \\
\hline & & B & 1 & 0 & 0 & 0 & & & & \\
\hline & 2 & $\mathrm{~A}$ & 1 & 1 & 0 & 0 & A & $\mathrm{B}$ & B & - \\
\hline & & B & 1 & 0 & 1 & 1 & & & & \\
\hline & 3 & $\mathrm{~A}$ & 1 & 1 & 0 & 0 & $\mathrm{~A}$ & $\mathrm{~A}$ & Guess & - \\
\hline & & $\mathrm{B}$ & 1 & 0 & 1 & 0 & & & & \\
\hline \multirow[t]{10}{*}{ IV } & 1 & A & 1 & 1 & 0 & 0 & A & A & A & A \\
\hline & & $\mathrm{B}$ & 1 & 0 & 0 & 0 & & & & \\
\hline & 2 & $\mathrm{~A}$ & 1 & 1 & 0 & 0 & $\mathrm{~A}$ & $\mathrm{~B}$ & B & B \\
\hline & & $\mathrm{B}$ & 1 & 0 & 1 & 1 & & & & \\
\hline & 3 & $\mathrm{~A}$ & 1 & 1 & 0 & 0 & $\mathrm{~A}$ & $\mathrm{~A}$ & Guess & $\mathrm{A}$ \\
\hline & & B & 1 & 0 & 1 & 0 & & & & \\
\hline & 4 & A & 1 & 1 & 0 & 0 & A & $\mathrm{A}$ & Guess & B \\
\hline & & $\mathrm{B}$ & 1 & 0 & 0 & 1 & & & & \\
\hline & 5 & $\mathrm{~A}$ & 1 & 0 & 0 & 1 & $\mathrm{~A}$ & $\mathrm{~B}$ & B & $\mathrm{A}$ \\
\hline & & B & 0 & 1 & 1 & 1 & & & & \\
\hline
\end{tabular}

$\mathrm{TTB}=$ take-the-best, $\mathrm{WADD}=$ weighted additive rule, $\mathrm{EQW}=$ equal weight rule, $\mathrm{TTS}=$ take the most salient, $\mathrm{CV}=$ congruent verbal condition, $\mathrm{IV}=$ incongruent verbal condition.

\section{Procedure}

The procedure was adopted from Bröder and Schiffer (2003, 2006b) and aligned to the SMC. The experiment consisted of three phases: a cue learning phase, a decision phase, and a final memory test.

Cue learning phase Participants learned the cue values of each of ten suspects by heart via an anticipation learning paradigm. Each suspect was identified by her name and a portrait. Names and portraits were randomly assigned to cue patterns. On each trial, the name and the portrait of one suspect were presented along with the verbal descriptions of the 12 possible cue values, grouped by cue. The cues were presented at different positions on screen (top, bottom, left, or right). The position of each cue was assigned randomly at the beginning of the experiment but was constant across trials (Fig. 1). For each cue, participants had to guess (recall) the correct cue value, starting with the most valid cue, and so on. After participants had clicked on a cue value, they received feedback about whether their answer was correct or not. Every suspect had to be repeated until all cue values were recalled correctly. These practice trials were continued until the cue values of all suspects had been learned that way. To ensure that participants had memorized the information thoroughly, these practice trials were followed by test trials. On the test trials, all suspects were presented consecutively, regardless of any errors in recall. Participants had to correctly recall at least $90 \%$ of the cue values to continue with the experiment. Otherwise the practice trials were repeated. The order of suspects and the order of cue values within each cue were determined randomly. After cue values had been learned, we informed participants about the predictive validity and the critical cue values of each cue.

Decision phase On each trial of the subsequent decision phase, the portraits and names of two suspects were presented in the middle of the screen, and participants had to decide which suspect was more likely to have committed the murder. The position of each suspect on screen was determined at random. Information about the cue values and the cue validities was never shown, but had to be retrieved from memory.

Additionally, four fields were shown at the same positions as the cues during the learning phase (top, bottom, left, and right). On each trial, one of the fields was colored purple, whereas the other white fields were just marked by a light purple frame (Fig. 2). By highlighting one of the fields, we guided a person's attention to a certain position on screen. According to the SMC, cue information that had been learned at this position before should be retrieved more easily than other cue information. Participants were told that the fields were irrelevant for making the decision and could therefore be ignored.

Participants had to make 73 decisions in randomized order, with the only exception being that identical paired comparisons were not presented consecutively. The number of trials resulted from a full paired comparison between the ten suspects (45 trials). Eight comparisons that discriminated between TTB and the compensatory strategies (WADD, 


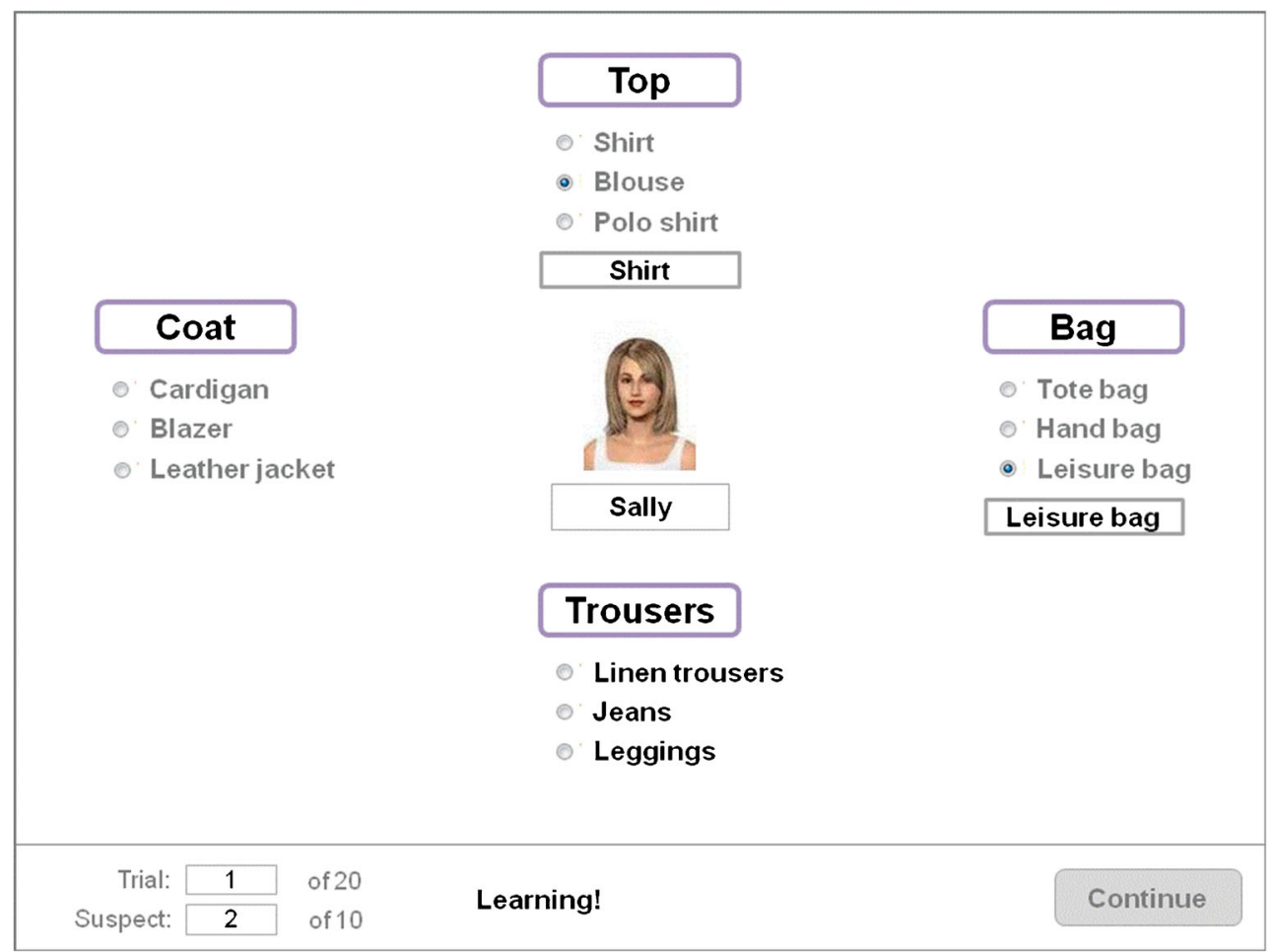

Fig. 1 Example of a trial in the cue learning phase in Experiments 1 and 2

EQW) were presented twice, to allow for a more reliable strategy classification. In all of these 53 trials, the most valid cue was highlighted by a purple field in the $\mathrm{CV}$ condition, whereas the least valid cue was highlighted in the IV condition. The strategy classification was based on the decisions made on these trials.

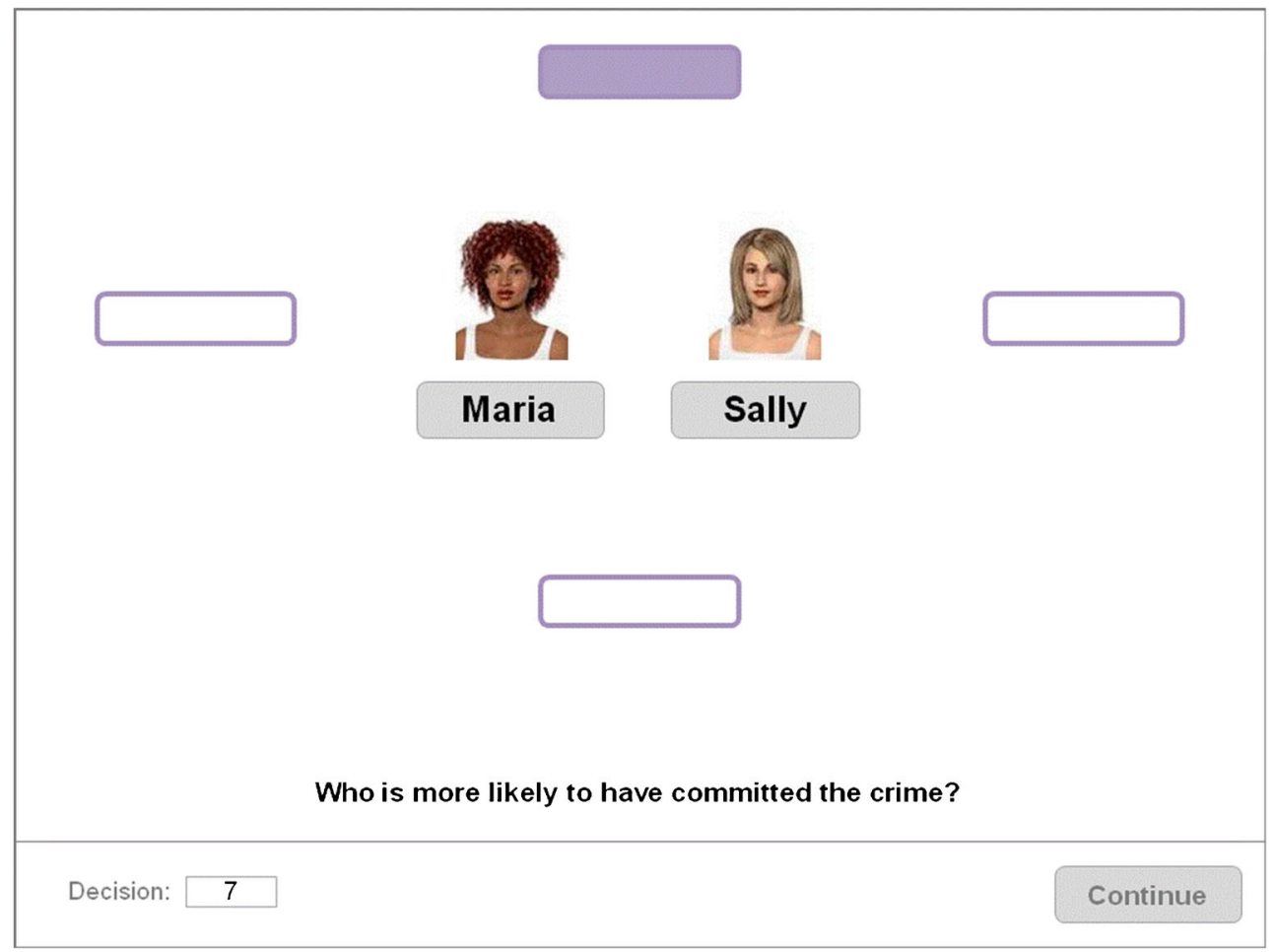

Fig. 2 Example of a trial in the decision phase in Experiments 1 and 2 
To reduce the demand characteristics of the manipulation, the 20 paired comparisons in which all strategies made identical predictions were presented a second time (see Table 2, Item Type 1). On these noncritical trials, the four positions were highlighted five times each. On two out of these five trials (about $11 \%$ of all trials), we introduced a recall test. After participants had made a decision, we asked them to recall one of the cue values that they had learned at the highlighted position before.

Final memory test One prerequisite for strategy classification is that cue values be remembered correctly throughout the experiment. To test memory performance, we conducted a memory test at the end of the experiment. The final memory test was identical in procedure to the test trials from the cue learning phase.

\section{Results}

Memory performance On average, $86.38 \%$ ( $S D=9.59)$ of the cue information was recalled correctly in the final memory test. Hence, the cue patterns in all experimental conditions were memorized well enough to warrant further analyses. As expected, cues did not differ with respect to their encoding quality. All four cues were remembered equally well $[F(3$, $189)=0.61, p=.61, \eta^{2}=.01,(1-\beta)=.99$, for a medium effect], and no interaction occurred for memory performance across cues and experimental conditions $[F(3,189)=0.88$, $p=.45, \eta^{2}=.01,(1-\beta)=.99$ for a medium effect $]$.

Classification of decision strategies We used an outcomebased maximum-likelihood method to assess the individual decision strategy (Bröder, 2010). In a nutshell, this method identifies the best-fitting strategy by comparing the predicted choice vector of each decision strategy with the observed choice vector of each participant. Four strategies were considered for classification: TTB, WADD, EQW, and a strategy that was referred to as "take the most salient" (TTS; Platzer \& Bröder, 2012). Platzer and Bröder conceptualized TTS as a noncompensatory strategy that searches cues sequentially, ordered by their visual salience. In the present experiment, however, either the most valid cue (CV condition) or the least valid cue (IV condition) was highlighted. The accessibility of all other cues was not manipulated. Hence, it would have been inadequate to fit a TTS strategy that was based on a distinct accessibility hierarchy of cues. Accordingly, we fitted a version in which TTS searches cues sequentially, starting with the cue that is easy to access (which is the most valid cue in the $\mathrm{CV}$ condition and the least valid cue in the IV condition). If this cue discriminates between options, TTS stops information search and chooses the option with the critical cue value. If this cue does not discriminate, TTS continues the information search according to the cue validities. Since TTB and TTS made identical predictions in the CV condition, TTS was fitted only in the IV condition. Assuming that individuals sometimes made errors when applying a strategy, binomial response error models served as a basis for classification. Participants were classified as being a user of the strategy with the highest likelihood if two conditions were met: First, the adherence rates for the best-fitting model should be higher than $60 \%$ (Bröder \& Schiffer, 2003). ${ }^{2}$ Secondly, the estimated response error $\widehat{\varepsilon}$ for the bestfitting model should be equally distributed across item types (see Table 2 for an overview of the different item types in the CV and IV conditions). Otherwise, the assumption that $\widehat{\varepsilon}$ reflects unsystematic error would be violated (Moshagen \& Hilbig, 2011). The pattern of a participant was classified as a guessing strategy if at least one requirement was not fulfilled. However, "guessing" included not only pure guessing, but also other strategies that we did not consider.

We report two different measures of classification reliability: likelihood ratios as a comparative measure, and adherence rates as a measure of absolute model fit. Likelihood ratios, denoting how much more likely the data are under the assumption of the best compared to the second-best model, indicated that more than $85 \%$ of all participants could be classified with at least moderate confidence (Wasserman, 2000; see our Table 3). ${ }^{3}$ Adherence rates indicated that across all conditions and strategies, about $80 \%$ of the observed choices matched the choices predicted by the classified strategy (Table 4).

Table 5 reports the strategy classification frequencies across conditions. More participants relied on TTB in the CV condition (58.82\%) than in the IV condition (25.81\%). In contrast, compensatory strategies were used more frequently in the IV condition (35.48\%) than in the CV condition (23.53\%, Fig. 3). Additionally, no participant used

\footnotetext{
${ }^{2}$ We used the convention introduced by Bröder and Schiffer (2003): Stricter criteria yield comparable effect sizes. The difference would still be conventionally significant with an adherence rate of $70 \%\left[\chi^{2}(1, N=\right.$ $41)=4.78, p=.03, \widehat{w}=.34]$, but not with an $80 \%$ threshold $\left[\chi^{2}(1, N=\right.$ $21)=2.65, p=.10, \widehat{w}=.36]$. Note the extremely low power in the latter case, however

${ }^{3}$ Why are the likelihood ratios for WADD and EQW smaller than the likelihood ratio of TTB? WADD and EQW can only be discriminated on the basis of some trials on which WADD makes distinct predictions, whereas EQW has to guess. Hence, the likelihoods of WADD and EQW are generally more similar than the likelihoods of TBB and either WADD or EQW.
} 
Table 3 Likelihood ratios (LR) for different strategies in Experiment 1 (strategy with highest likelihood divided by strategy with secondhighest likelihood)

\begin{tabular}{llllll}
\hline & & \multicolumn{2}{l}{$\%$ of Participants With Different LRs } & \\
\cline { 4 - 6 } Strategy & $N$ & Md (LR) & LR $<3$ (weak evidence) & $3<$ LR $\leq 10$ (moderate evidence) & LR $>10$ (strong evidence) \\
\hline TTB & 28 & 125.48 & - & 28.57 & 71.43 \\
WADD & 10 & 6.92 & 30.00 & 30.00 & 40.00 \\
EQW & 9 & 13.01 & 44.44 & 11.11 & 44.44 \\
\hline
\end{tabular}

$\mathrm{TTB}=$ take-the-best, $\mathrm{WADD}=$ weighted additive rule, $\mathrm{EQW}=$ equal weight rule.

${ }^{a}$ Conventions for weak/moderate/strong evidence in favor of a model are based on Wasserman (2000).

the TTS strategy. Comparing the frequency distributions of TTB and the pooled compensatory strategies (COMP: i.e., WADD and EQW) across experimental conditions indicated a preponderance of TTB in the CV condition, whereas most participants used compensatory strategies in the IV condition $\left[\left(\chi^{2}(1, N=47)=4.04, p=.04, \widehat{w}=0.29\right]\right.$.

\section{Discussion}

The results of Experiment 1 corroborate the assumption that the accessibility effect also occurs with verbal materials and if accessibility is manipulated during retrieval. Hence, the salience effect is a special case of a broader phenomenon that is based on the accessibility of cue information in memory. Experiment 1 replicated Platzer and Bröder's (2012) result that strategy selection is influenced by the interaction between the validity and the accessibility of cue information. As we hypothesized, participants relied on TTB if valid information came to mind easily. Here, involuntary retrieval of highly accessible information would not interfere with TTB's deliberate search order, but rather would support it. However, if less valid information came to mind easily, neither was this information ignored (by also using TTB) nor did people decide solely on the basis of this information (by using TTS). Rather, most participants relied on some kind of compensatory strategy in the IV condition. Additionally, the number of guessers increased in the IV relative to the $\mathrm{CV}$ condition $\left[\left(\chi^{2}(2, N=64)=6.86, p=\right.\right.$

Table 4 Adherence rates of decision strategies in Experiment 1

\begin{tabular}{llll}
\hline \multirow{4}{*}{ Condition } & \multicolumn{2}{l}{ Decision Strategy } & \\
\cline { 2 - 4 } & TTB & WADD & EQW \\
\hline CV & 77.08 & 75.85 & 87.04 \\
IV & 74.06 & 86.04 & 82.87 \\
\hline
\end{tabular}

$\mathrm{TTB}=$ take-the-best, WADD $=$ weighted additive rule, $\mathrm{EQW}=$ equal weight rule, $\mathrm{CV}=$ congruent verbal condition, $\mathrm{IV}=$ incongruent verbal condition. Adherence rates are based on the participants classified as using this strategy.
$.03, \widehat{w}=0.33]$. One plausible explanation is based on the finding that simple heuristics, such as TTB, are used more frequently if decisions have to be made from memory, in order to keep mental costs as low as possible (Bröder \& Schiffer, 2003). Using TTB in the IV condition would require ignoring information that came to mind easily and concentrating on valid information instead. In fact, it took TTB users longer to complete the decision phase in the IV condition $(18.03 \mathrm{~min})$ than in the $\mathrm{CV}$ condition (11.98 $\min )[t(26)=2.08, p=.05, d=0.87]$ namely, approximately as long as it took compensatory decision makers $(21.88 \mathrm{~min})$ in both conditions $[t(25)=0.37, p=.72, d=0.15,(1-\beta)=.21$, for a medium effect]. If for a person both TTB and COMP were too cognitively demanding, the only alternative might have been just to guess, since in general TTS was not considered appropriate.

One reason for developing the SMC was the ambition to investigate accessibility effects that are independent of the availability of cue information. Therefore, we manipulated the accessibility of cues exclusively during the decision phase. This was done to ensure that accessible cues were not memorized better in the learning phase than were less accessible cues. Our results concerning memory performance for cues could be interpreted as a measure of the discriminant validity of the experimental manipulation. All cues were remembered equally well, regardless of our experimental manipulation. Hence, the SMC did not influence the availability of information in memory.

Table 5 Frequencies and percentages of decision strategies (in parentheses) within conditions in Experiment 1

\begin{tabular}{|c|c|c|c|c|c|}
\hline \multirow[b]{2}{*}{ Condition } & \multicolumn{5}{|c|}{ Decision Strategy } \\
\hline & ТTВ & WADD & EQW & Guess & Unclass \\
\hline $\mathrm{CV}$ & $20(58.82)$ & $5(14.71)$ & $3(8.82)$ & $6(17.65)$ & - \\
\hline IV & $8(25.81)$ & $5(16.13)$ & $6(19.35)$ & $11(35.48)$ & $1(3.23)$ \\
\hline
\end{tabular}

$\mathrm{TTB}=$ take-the-best, WADD $=$ weighted additive rule, $\mathrm{EQW}=$ equal weight rule, Guess = guessing (adherence rates $<60 \%$ and/or errors not randomly distributed across item types), Unclass $=$ unclassified pattern (identical likelihoods for two strategies), $\mathrm{CV}=$ congruent verbal condition, IV = incongruent verbal condition. 


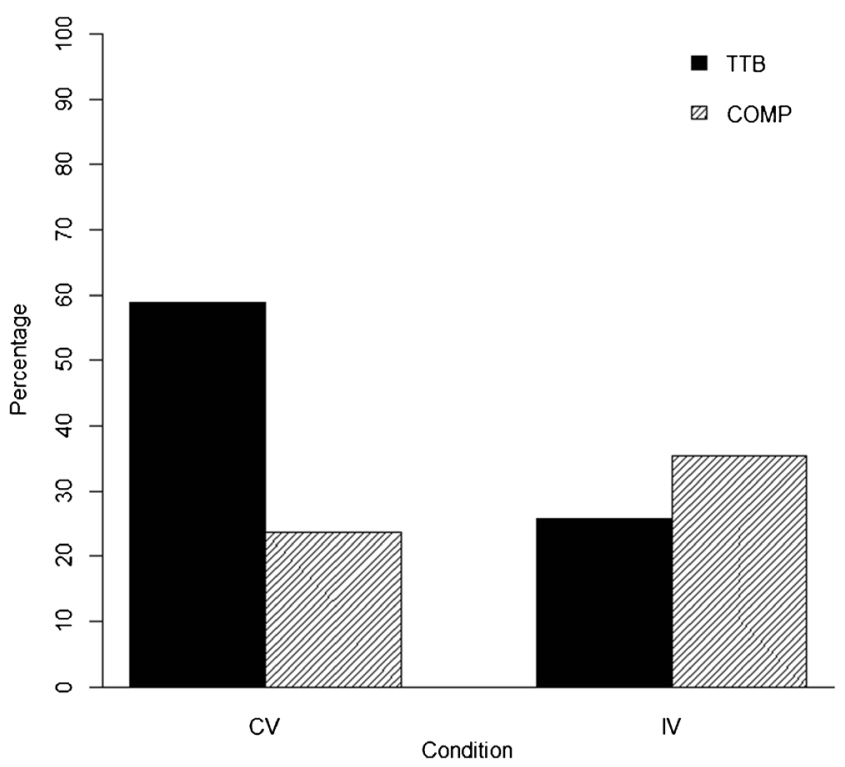

Fig. 3 Percentages of participants using take-the-best (TTB) or a compensatory strategy (COMP: WADD or EQW) in Experiment 1, depending on experimental conditions ( $\mathrm{CV}$, congruent verbal; IV, incongruent verbal)

\section{Experiment 2: Manipulating accessibility in a within-subjects design}

In Experiment 1, accessibility was manipulated in a betweensubjects design. The results indicated that people applied different decision strategies, depending on whether the most valid cue (CV condition) or the least valid cue (IV condition) could be accessed easily.

In principle, strategy classification methods are based on the presupposition that decision makers use a certain strategy throughout the experiment. Choosing the option that is not predicted by the applied strategy is attributed to unsystematic response error. In Experiment 2, these changes in the decision behavior of participants were precisely what we were interested in. For Experiment 2, we aimed at replicating the results from Experiment 1 by means of a within-subjects design. In this experiment, we were not interested in classifying participants as users of a certain strategy. Rather, we investigated whether the decision behavior changed throughout the decision process, depending on the validity of easily accessible cues. If this was the case, we would learn something on a theoretical (1) as well as on a methodological (2) level: (1) If people were to adapt their decision behavior on a trial-by-trial level, this would mean that they were highly sensitive to the accessibility of information. Not only would they establish a certain strategy, but they could adapt their decision behavior when the validity of easily accessible cues changed. This would be particularly interesting for research on decision routines, which has suggested that people are extremely reluctant to change their decision strategy (e.g., Bröder \& Schiffer, 2006a). More importantly, however, such a result would perhaps put in question the idea that strategies are selected in the first place. (2) On a methodological level, short-term changes in decision behavior according to the cues' accessibility could be interpreted in terms of the construct validity of our experimental manipulation: If decision behavior can be manipulated on a trial level with the SMC, this would provide further evidence that the SMC actually manipulates short-term accessibility.

\section{Method}

Participants

A group of 76 students from the University of Mannheim participated in Experiment 2 (55 females, 21 males; mean age 21 years, $S D=2.50)$. One participant had to be excluded because of an attempt to deceive. ${ }^{4}$ The participants received either course credit or $€ 5$ per hour, plus a performancecontingent payment.

\section{Materials and design}

We used the same cover story of an invented criminal case as in Experiment 1. All of the materials used in the Experiments 1 and 2 were identical. In contrast to Experiment 1, cue accessibility was manipulated in a within-subjects design. On half of the critical trials, the most valid cue was made easily accessible (congruent verbal, CV). On the other half of the trials, the least valid cue was made easily accessible (incongruent verbal, IV).

\section{Procedure}

As in Experiment 1, the procedure was subdivided into three phases: (1) In the cue learning phase, the cue values of each suspect had to be learned by means of an anticipation learning paradigm. (2) In the subsequent decision phase, participants had to decide which suspect was more likely to have committed the murder by retrieving information about the cue values and validities from memory. (3) Memory performance for the cue values was tested in the final memory test. The procedures of Experiments 1 and 2 were identical, except for the composition of the trials in the decision phase. Therefore, we will only describe the decision phase in greater detail.

Decision phase Participants had to make 53 decisions in randomized order, with the only exception being that identical paired comparisons were not presented consecutively. The

\footnotetext{
${ }^{4}$ The participant avoided learning the cue patterns during the learning phase by taking pictures of the suspects with a cellphone.
} 
eight decisions that discriminated between TTB and either WADD or EQW were presented twice. On four of these critical trials (which were presented twice), the most valid cue was highlighted by the purple field (CV trials). On the remaining four critical trials (again presented twice), the least valid cue was highlighted (IV trials). All of the following analyses are based on these 16 critical trials, since all strategies made the same predictions on the remaining 38 trials. On these 38 trials, the fields were highlighted such that across all 53 trials, every position was highlighted equally often. The recall test was accomplished only after these noncritical trials.

\section{Results}

\section{Memory performance}

With $88.27 \%(S D=7.93)$ of the cue information being recalled correctly, memory performance was comparably high relative to that in Experiment 1. Additionally, all cues were remembered equally well $[F(3,222)=2.59, p=.054$, $\eta^{2}=.03,(1-\beta)=.99$, for a medium effect $]$.

\section{Decision behavior}

In Experiment 2, cue accessibility was manipulated on a trial level. By highlighting one of four positions on screen, we enhanced the accessibility of the cue information that had been learned at this position. As was hypothesized, the TTB option was chosen more often if the most valid cue was easily accessible $(53.83 \%$ of all critical CV trials). In contrast, participants were less likely to choose the TTB option-and, complementarily, they were more likely to choose the compensatory option - if the least valid cue was easily accessible $[45.50 \%$ of all critical IV trials; mean difference $=$ 8.33, $S E$ (mean difference) $=3.82, p=.03, d=0.36$, on the basis of bootstrap results with 3,000 samples]. ${ }^{5}$

\section{Discussion}

The results of Experiment 2 support the finding that the validity as well as the accessibility of cue information is

\footnotetext{
${ }^{5}$ We also conducted the same analyses for Experiment 1 . In line with Experiment 2, participants were more likely to choose the option predicted by TTB in the CV condition ( $60.11 \%$ of all critical trials) than in the IV condition ( $45.16 \%$ of all critical trials). However, the difference was not significant on the basis of bootstrap results with 3,000 samples [mean difference $=14.95, S E$ (mean difference $)=7.48, p=.051$, $d=0.50]$, which might be due to low statistical power.
}

important to explain decision behavior: People were more likely to choose the option predicted by TTB if the accessibility and validity of a cue converged. If easily accessible information was invalid, people preferred to choose the option consistent with WADD or EQW. This was demonstrated in a within-subjects design in which accessibility was manipulated on a trial level: On half of the critical trials, the most valid cue was highlighted. On the other half of the critical trials, the least valid cue was highlighted.

Our results indicate that people react highly sensitively to the accessibility of information. They change their decision behavior even within one task, depending on the validity of the easily accessible information. The finding that decision behavior can be manipulated on a trial level with the SMC provides further evidence that it actually manipulates shortterm accessibility.

\section{General discussion}

Showing that judgment and decision behavior is influenced by the accessibility of information in memory has a long tradition (see e.g., Marewski \& Schooler, 2011). It has been shown that the ease with which information can be brought to mind affected, for instance, the estimated frequencies and probabilities of events (availability heuristic; e.g., Gabrielcik \& Fazio, 1984; Lichtenstein, Slovic, Fischhoff, Layman, \& Combs, 1978; Pachur, Hertwig, \& Steinmann, 2012; Tversky \& Kahneman, 1973), judgments about the self and others (e.g., Jacoby, Kelley, Brown, \& Jasechko, 1989; Schwarz et al., 1991), judgments of truth (Reber \& Schwarz, 1999), and recognition judgments (e.g., Johnston, Dark, \& Jacoby, 1985; Johnston, Hawley, \& Elliott, 1991). Moreover, manipulating the processing fluency of certain features in multi-cue tasks influenced the evaluations of objects (Shah \& Oppenheimer, 2007) and also object preferences (Mandel \& Johnson, 2002).

The experiments reported in this article took a closer look at the cognitive processes underlying decision behavior in multi-cue inferences from memory. This article aimed at answering the question whether-and, if so, howindividuals consider easily accessible information, depending on the predictive validity of this information. Platzer and Bröder (2012) were the first to show that strategy selection depends on the interaction between the accessibility and the validity of cues. In their study, accessibility was manipulated via the visual salience of pictorial cues. We developed the SMC to test whether their findings can be replicated (1) with verbal materials and (2) if accessibility is manipulated during retrieval.

In the introduction, we asked three questions to which our study could provide answers: (1) Do people ignore easily accessible information that is not valid? If this was true, we 
would expect to find the same amount of TTB users (Exp. 1) or TTB-compatible choices (Exp. 2) in the incongruent and congruent conditions. Clearly, this was not the case. As compared to the congruent conditions, fewer participants used TTB in the incongruent conditions. Reaction time data indicated that it seemed to be equally cognitively demanding to ignore invalid information if it came to mind easily as to integrate it into the judgment (cf., Platzer \& Bröder, 2012). In a nutshell, the results indicate that accessible information is considered. Presumably, involuntary retrieval interferes with the deliberate search order of TTB. The second and the third questions addressed the issue whether accessible information is integrated in a noncompensatory, or rather compensatory, manner. (2) Do people exclusively rely on information that can be accessed easily, regardless of its validity? In this case, we would expect to find a considerable number of TTS users in the incongruent condition. This was not confirmed. No single person used TTS in Experiment 1. (3) Or, is information that can be accessed easily just more likely to be incorporated into the judgment in a compensatory manner? In line with previous studies, our results suggest that this was the case. Individuals did not trust information only because it could be easily accessed. Rather, they integrated all available cue information in a compensatory manner if invalid information came to mind easily. Since this also happened on a trial-by-trial basis in Experiment 2's within subjects manipulation, one may speculate that an evidence accumulation model (e.g., Lee \& Cummins, 2004) rather than a model assuming strategy selection is more appropriate. In such a model, the weighting of cues could be a function of both validity and salience of cues. However, our results can be interpreted in both frameworks.

From an evolutionary perspective, it might be considered advantageous to take easily accessible information into account. Fine and Minnery (2009) argued that " $[t]$ he tendency to orient attention toward visually salient stimuli is conserved across species and likely confers an evolutionary advantage by enabling an organism to rapidly detect and react to behaviorally relevant objects and events within its environment" (p. 8018). This idea was implemented also in early theories of associative learning, which pointed to the relation between stimulus salience and "associability": Salient stimuli enter into associations more readily than do other stimuli (e.g., Rescorla, 1988). Studies within the context of cognitive fluency also suggested an ecological correlation between retrieval fluency (e.g., manipulated via visual salience; Unkelbach, 2006) and the validity of information (Hertwig, Herzog, Schooler, \& Reimer, 2008), and that in certain environments, a strategy that orders cues according to the speed of retrieving them could make good predictions (Gaissmaier, 2007). Although it seems to be adaptive to consider salient information in some natural environments, one can imagine several counterexamples, especially in the field of sales promotion. Going back to our example of buying healthy cereals: Would it be a good idea to buy oat cereal just because the first thing that comes to your mind is the goodlooking organic farmer from the billboard?

People might incorporate easily accessible information because they believe in this positive correlation between the accessibility of information and its validity. For instance, Shah and Oppenheimer (2007) provided evidence that people placed more weight on information that felt easy to process. As a consequence, people should tend to overestimate the importance of invalid cues that can be accessed easily. We tested this assumption empirically by asking participants to evaluate the importance of each cue for the prediction of the decision criterion at the end of Experiment 1. Importance ratings were judged per cue using a slider that ranged from 0 (unimportant) to 100 (very important). If the accessibility of a cue affected its perceived importance, we would expect participants to report higher subjective importance ratings for less valid cues in the incongruent than in the congruent condition. Hence, we would expect the structure of cue weights to be more compensatory in the IV than in the CV condition. This assumption was not supported by our data. A repeated measures analysis of variance with the Importance Ratings for each of the four cues as a within-subjects factor and Condition as a between-subjects factor did not provide any evidence for an interaction between cue ratings and condition $\left[F(2.06,129.79)=0.30, p=.75, \eta^{2}=.005\right.$, $(1-\beta)=.99$, for a medium effect]. Additionally, the variances of importance ratings of the four cues did not differ between conditions, which should have been the case if we expected a more compensatory structure of ratings in the IV condition $[$ mean difference $=126.98, S E($ mean difference $)=$ $159.89, p=.44, d=0.20$, on the basis of bootstrap results with 3,000 samples]. In sum, these results indicate that people probably did not (mis)attribute the higher accessibility of a cue to its validity, as was suggested by Shah and Oppenheimer (2007) and Oppenheimer (2008). One possible explanation for these diverging results is that Shah and colleagues did not provide cue validities. As a consequence, processing fluency was the only basis for inferring the importance of each cue. Since this explanation is post-hoc, it will be an interesting avenue for future research to further disentangle the interaction between the accessibility and the validity of cue information. Maybe one can think of constellations of cues in which the accessibility overrides the validity of cues. For instance, one might expect to find TTS users if the cues did not differ in validity. Additionally, this might also affect importance ratings, since there would be no other clue for ordering the cues, as in the studies by Shah and Oppenheimer.

One caveat about all studies on memory-based probabilistic inferences hitherto conducted concerns the boundary conditions of the effects reported. In these studies, cue information of objects had been learned just prior to the decision phase, which might lead to a memory representation 
quite different from, for example, the well-integrated, or even automatized, knowledge of experts. Whether the formerly reported effects of retrieval costs and their manipulation conducted here would generalize to situations involving more expertise is ultimately an interesting empirical question yet to be researched.

Given the results reported here, does it make sense from a marketing perspective to make certain positive characteristics of a product particularly accessible, even if they do not correlate with the criterion dimension? For example, if you want people to believe that your cereals are exceptionally healthy, does it make sense to let an eye-catching organic farmer-who will easily be remembered later-smile at pedestrians from a billboard? The answer is "yes" and "no." By doing so, you can make sure that this information will be considered during the decision process, especially if it is invalid. Nevertheless, you cannot count on people deciding solely on the basis of this information.

Open Access This article is distributed under the terms of the Creative Commons Attribution License which permits any use, distribution, and reproduction in any medium, provided the original author(s) and the source are credited.

\section{References}

Altmann, G. T. M. (2004). Language-mediated eye movements in the absence of a visual word: The "blank screen paradigm. Cognition, 93, B79-B78.

Beach, L. R., \& Mitchell, T. R. (1978). A contingency model for the selection of decision strategies. Academy of Management Review, 3 , 439-449.

Bröder, A. (2000). Assessing the empirical validity of the "Take-the-best" heuristic as a model of human probabilistic inference. Journal of Experimental Psychology: Learning, Memory, and Cognition, 26, 1332-1346. doi:10.1037/0278-7393.26.5.1332

Bröder, A. (2003). Decision making with the "Adaptive Toolbox": Influence of environmental structure, intelligence, and working memory load. Journal of Experimental Psychology: Learning, Memory, and Cognition, 29, 611-625. doi:10.1037/0278-7393.29.4.611

Bröder, A. (2010). Outcome-based strategy classification. In A. Glöckner \& C. L. M. Witteman (Eds.), Foundations for tracing intuition: Challenges and methods (pp. 61-82). London, UK: Psychology Press.

Bröder, A., \& Schiffer, S. (2003). "Take the best" versus simultaneous feature matching: Probabilistic inferences from memory and effects of representation format. Journal of Experimental Psychology: General, 132, 277-293. doi:10.1037/0096-3445.132.2.277

Bröder, A., \& Schiffer, S. (2006a). Adaptive flexibility and maladaptive routines in selecting fast and frugal decision strategies. Journal of Experimental Psychology: Learning, Memory, and Cognition, 32, 904-918.

Bröder, A., \& Schiffer, S. (2006b). Stimulus format and working memory in fast and frugal strategy selection. Journal of Behavioral Decision Making, 19, 361-380.

Chandon, P., \& Wansink, B. (2002). When are stockpiled products consumed faster? A convenience-salience framework of postpurchase consumption incidence and quantity. Journal of Marketing Research, 34, 321-335.
Dougherty, M. R. P., Gronlund, S. D., \& Gettys, C. F. (2003). Memory as a fundamental heuristic for decision making. In S. L. Schneider \& J. Shanteau (Eds.), Emerging perspectives on judgment and decision making (pp. 125-164). Cambridge, UK: Cambridge University Press.

Egeth, H. E., \& Yantis, S. (1997). Visual attention: Control, representation, and time course. Annual Review of Psychology, 48, 269-297. doi:10.1146/annurev.psych.48.1.269

Ferreira, F., Apel, J., \& Henderson, J. M. (2008). Taking a look at looking at nothing. Trends in Cognitive Sciences, 12, 405-410. doi:10.1016/ j.tics.2008.07.007

Fine, M. S., \& Minnery, B. S. (2009). Visual salience affects performance in a working memory task. Journal of Neuroscience, 24, 8016-8021.

Gabrielcik, A., \& Fazio, R. H. (1984). Priming and frequency estimation: A strict test of the availability heuristic. Personality and Social Psychology Bulletin, 10, 85-89.

Gaissmaier, W. (2007). The mnemonic decision maker: How search in memory shapes decision making. $\mathrm{PhD}$ dissertation, Freie Universität Berlin, Germany.

Gigerenzer, G., \& Goldstein, D. G. (1996). Reasoning the fast and frugal way: Models of bounded rationality. Psychological Review, 103, 650-669. doi:10.1037/0033-295X.103.4.650

Gigerenzer, G., Hoffrage, U., \& Kleinbölting, H. (1991). Probabilistic mental models: A Brunswikian theory of confidence. Psychological Review, 98, 506-528. doi:10.1037/0033-295X.98.4.506

Gigerenzer, G., \& Todd, P. M. (1999). Fast and frugal heuristics: The adaptive toolbox. In G. Gigerenzer, P. M. Todd, \& the ABC Research Group (Eds.), Simple heuristics that make us smart (pp. 3-34). New York, NY: Oxford University Press.

Hastie, R., \& Park, B. (1986). The relationship between memory and judgment depends on whether the judgment task is memory-based or on-line. Psychological Review, 93, 258-268.

Hertwig, R., Herzog, S. M., Schooler, L. J., \& Reimer, T. (2008). Fluency heuristic: A model of how the mind exploits a by-product of information retrieval. Journal of Experimental Psychology: Learning, Memory, and Cognition, 34, 1191-1206.

Itti, L., \& Koch, C. (2000). A saliency-based search mechanism for overt and covert shifts of visual attention. Vision Research, 40, 14891506. doi:10.1016/S0042-6989(99)00163-7

Itti, L., \& Koch, C. (2001). Computational modeling of visual attention. Nature Reviews Neuroscience, 2, 194-203. doi:10.1038/35058500

Jacoby, L. L., Kelley, C., Brown, J., \& Jasechko, J. (1989). Becoming famous overnight: Limits on the ability to avoid unconscious influences of the past. Journal of Personality and Social Psychology, 56, 326-338.

James, W. (1890). The principles of psychology. New York, NY: Holt.

Johansson, R. (2013, March). Eye movements to "nothing" have an active role during visuospatial memory retrieval. Paper presented at the 55th Conference of Experimental Psychologists, TeaP, Vienna, Austria.

Johnston, W. A., Dark, V. J., \& Jacoby, L. L. (1985). Perceptual fluency and recognition judgments. Journal of Experimental Psychology: Learning, Memory, and Cognition, 11, 3-11. doi:10.1037/0278-7393.11.1.3

Johnston, W. A., Hawley, K. J., \& Elliott, J. M. G. (1991). Contribution of perceptual fluency to recognition judgments. Journal of Experimental Psychology: Learning, Memory, and Cognition, 17, 210-223. doi:10.1037/0278-7393.17.2.210

Kardes, F. R. (1999). Consumer behavior and managerial decision making. Reading, MA: Addison-Wesley.

Lee, M. D., \& Cummins, D. R. (2004). Evidence accumulation in decision making: Unifying the "take the best" and the "rational" models. Psychonomic Bulletin \& Review, 11, 343-352.

Lichtenstein, S., Slovic, P., Fischhoff, B., Layman, M., \& Combs, B. (1978). Judged frequency of lethal events. Journal of Experimental Psychology: Human Learning and Memory, 4, 551-578.

Logan, G. D. (1988). Toward an instance theory of automatization. Psychological Review, 95, 492-527. doi:10.1037/0033-295X.95.4.492 
Mandel, N., \& Johnson, E. J. (2002). When web pages influence choice: Effects of visual primes on experts and novices. Journal of Consumer Research, 29, 235-245.

Marewski, J. N., \& Schooler, L. J. (2011). Cognitive niches: An ecological model of strategy selection. Psychological Review, 118, 393-437.

Moshagen, M., \& Hilbig, B. E. (2011). Methodological notes on model comparison and strategy classification: A falsificationist proposition. Judgment and Decision Making, 6, 814-820.

Newell, B. R., \& Shanks, D. R. (2003). Take the best or look at the rest? Factors influencing "one-reason" decision making. Journal of Experimental Psychology: Learning, Memory, and Cognition, 29, 53-65. doi:10.1037/0278-7393.29.1.53

Newell, B. R., Weston, N. J., \& Shanks, D. R. (2003). Empirical tests of a fast-and-frugal heuristic: Not everyone "takes-the-best. Organizational Behavior and Human Decision Processes, 91, 82-96.

Olson, G. D. (2002). Salient stimuli in advertising: The effect of contrast interval length and type on recall. Journal of Experimental Psychology: Applied, 8, 168-179.

Oppenheimer, D. (2008). The secret life of fluency. Trends in Cognitive Sciences, 12, 237-241.

Pachur, T., Hertwig, R., \& Steinmann, F. (2012). How do people judge risk: Availability heuristic, affect heuristic, or both? Journal of Experimental Psychology: Applied, 18, 314-330.

Parkhurst, D., Law, K., \& Niebur, E. (2002). Modeling the role of visual salience in the allocation of overt visual attention. Vision Research, 42, 107-123. doi:10.1016/S0042-6989(01)00250-4

Parkhurst, D. J., \& Niebur, E. (2004). Texture contrast attracts overt visual attention in natural scenes. European Journal of Neuroscience, 19, 783-789.

Pashler, H. (1988). Cross-dimensional interaction and texture segregation. Perception \& Psychophysics, 43, 307-318. doi:10.3758/BF03208800

Payne, J. W., Bettman, J. R., \& Johnson, E. J. (1988). Adaptive strategy selection in decision making. Journal of Experimental Psychology: Learning, Memory, and Cognition, 14, 534-552.

Payne, J. W., Bettman, J. R., \& Johnson, E. J. (1993). The adaptive decision maker. Cambridge, UK: Cambridge University Press.

Platzer, C., \& Bröder, A. (2012). Most people do not ignore salient invalid cues in memory-based decisions. Psychonomic Bulletin \& Review, 19, 654-661. doi:10.3758/s13423-012-0248-4

Posner, M. I. (1980). Orienting of attention. Quarterly Journal of Experimental Psychology, 32, 3-25. doi:10.1080/00335558008248231

Posner, M. I., Snyder, C. R. R., \& Davidson, B. J. (1980). Attention and the detection of signals. Journal of Experimental Psychology: General, 109, 160-174. doi:10.1037/0096-3445.109.2.160
Reber, R., \& Schwarz, N. (1999). Effects of perceptual fluency on judgments of truth. Consciousness and Cognition, 8, 338342.

Renkewitz, F., \& Jahn, G. (2012). Memory indexing: A novel method for tracing memory processes in complex cognitive tasks. Journal of Experimental Psychology: Learning, Memory, and Cognition, 38, 1622-1639. doi:10.1037/a0028073

Rescorla, R. A. (1988). Behavioral studies of Pavlovian conditioning. Annual Review of Neuroscience, 11, 329-352.

Richardson, D. C., \& Spivey, M. J. (2000). Representation, space and Hollywood squares: Looking at things that aren't there anymore. Cognition, 76, 269-295. doi:10.1016/S0010-0277 (00)00084-6

Rieskamp, J., \& Hoffrage, U. (1999). When do people use simple heuristics, and how can we tell? In G. Gigerenzer, P. M. Todd, \& the ABC Research Group (Eds.), Simple heuristics that make us smart (pp. 141-167). New York, NY: Oxford University Press.

Rieskamp, J., \& Hoffrage, U. (2008). Inferences under time pressure: How opportunity costs affect strategy selection. Acta Psychologica, 127, 258-276.

Rieskamp, J., \& Otto, P. E. (2006). SSL: A theory of how people learn to select strategies. Journal of Experimental Psychology: General, 135, 207-236. doi:10.1037/0096-3445.135.2.207

Schwarz, N., Bless, H., Strack, F., Klumpp, G., Rittenauer-Schatka, H., \& Simons, A. (1991). Ease of retrieval as information: Another look at the availability heuristic. Journal of Personality and Social Psychology, 61, 195-202.

Shah, A. K., \& Oppenheimer, D. M. (2007). Easy does it: The role of fluency in cue weighting. Judgment and Decision Making, 2, 371-379.

Theeuwes, J. (1991). Cross-dimensional perceptual selectivity. Perception \& Psychophysics, 50, 184-193. doi:10.3758/ BF03212219

Theeuwes, J. (1992). Perceptual selectivity for color and form. Perception \& Psychophysics, 51, 599-606. doi:10.3758/BF03211656

Tulving, E., \& Pearlstone, Z. (1966). Availability versus accessibility of information in memory for words. Journal of Verbal Learning and Verbal Behavior, 5, 381-391. doi:10.1016/S0022-5371(66)80048-8

Tversky, A., \& Kahneman, D. (1973). Availability: A heuristic for judging frequency and probability. Cognitive Psychology, 5, 207232. doi:10.1016/0010-0285(73)90033-9

Unkelbach, C. (2006). The learned interpretation of cognitive fluency. Psychological Science, 17, 339-345.

Wasserman, L. (2000). Bayesian model selection and model averaging. Journal of Mathematical Psychology, 44, 92-107. 